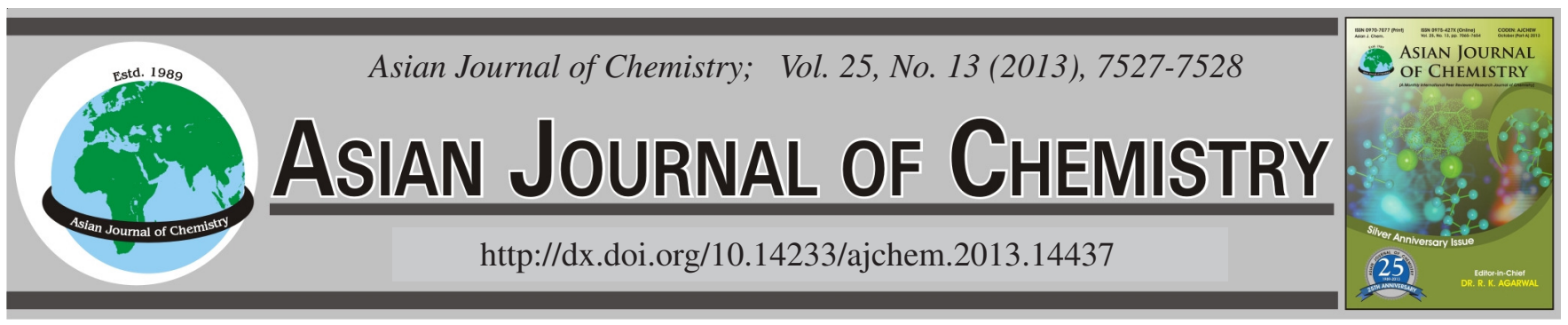

NOTE

\title{
Supramolecular Structure of 1-Phenyl-3-methyl-4-benzoylpyrazolon-5-one
}

Li Xu, Zheng-Kun Wang, Yin-XIA Sun ${ }^{*}$, Yu-Jie Zhang and Xiu-Yan Dong

School of Chemical and Biological Engineering, Lanzhou Jiaotong University, Lanzhou 730070, P.R. China

*Corresponding author: E-mail: sun_yinxia@163.com

(Received: 16 August 2012;

Accepted: 5 July 2013)

AJC-13767

The compound 1-phenyl-3-methyl-4-benzoylpyrazolon-5-one with the molecular formula $\mathrm{C}_{17} \mathrm{H}_{14} \mathrm{~N}_{2} \mathrm{O}_{2}$, exists in a keto-enamine tautomeric form. The pyrazolone ring makes dihedral angles of 28.36(3) and 59.31(5) $)^{\circ}$ with the two phenyl rings. The molecules are linked into twodimensional supramolecular structure by a combination of $\mathrm{N}-\mathrm{H} \cdots \mathrm{O}$ and $\mathrm{C}-\mathrm{H} \cdots \mathrm{O}$ hydrogen-bonding interactions.

Key Words: 1-Phenyl-3-methyl-4-benzoylpyrazolon-5-one, Supramolecular structure.

1-Phenyl-3-methyl-4-benzoylpyrazolon-5-one, an effective $\beta$-diketonate, is widely used and well known for its extractive ability ${ }^{1-3}$. In recent years, its metal complexes have also been found to have good antibacterial and biological properties. Its metal complexes have analgesic activity ${ }^{4-6}$. For our interest in super supramolecular chemistry, herein we've mainly studied the supramolecular structure of the this compound.

1-Phenyl-3-methyl-5-pyrazolone was purchased from Alfa Aesar and used without any purification. The reagents and solvents were analytical grade reagents from Tianjin Chemical Reagent Factory. C, H and N analyses were carried out with a GmbH VariuoEL V3.00 automatic elemental analyzer. Melting points were measured by the use of a microscopic melting point apparatus made in Beijing Taike Instrument Limited Company and the thermometer was uncorrected. X-Ray single crystal structure was determined on a Bruker Smart APEX CCD area detector.

General procedure: 1-Phenyl-3-methyl-5-pyrazolone $(3.75 \mathrm{~g})$ was dissolved in $80 \mathrm{~mL}$ of 1,4-dioxane by heating. Calcium hydroxide $(6 \mathrm{~g})$ was added followed by addition of benzoyl chloride $(3 \mathrm{~mL})$ dropwise within $1 \mathrm{~min}$. The temperature increased during the first few minutes and the reaction mixture became a thick paste. The reaction mixture was refluxed for $0.5 \mathrm{~h}$. Calcium complex of the desired ligand formed in the flask was decomposed by pouring the mixture into dilute hydrochloric acid $(50 \mathrm{~mL})$. An orange precipitate was formed slowly. It was filtered under suction, washed with a little water and 1,4-dioxane and recrystallised from a slightly acidified methanol-water mixture to destroy any undecomposed calcium complex. Yield $79.8 \%$. m.p. 89-91 ${ }^{\circ} \mathrm{C}$. Anal. calcd. (\%) for $\mathrm{C}_{17} \mathrm{H}_{14} \mathrm{~N}_{2} \mathrm{O}_{2}: \mathrm{C}, 73.25 ; \mathrm{H}, 5.04 ; \mathrm{N}, 10.01$. Found (\%): C, 73.37; H, 5.07; N, 10.07 .

Colourless needle-like single crystals suitable for X-ray diffraction studies were obtained by slow evaporation from a solution of dichloromethane at room temperature over a period of approximately one month.

\begin{tabular}{|c|c|}
\hline \multicolumn{2}{|c|}{$\begin{array}{l}\text { TABLE-1 } \\
\text { CRYSTAL DATA AND REFINEMENT PARAMETERS } \\
\text { FOR THE TITLE COMPOUND }\end{array}$} \\
\hline Empirical formula & $\mathrm{C}_{17} \mathrm{H}_{14} \mathrm{~N}_{2} \mathrm{O}_{2}$ \\
\hline Formula weight & 278.30 \\
\hline Temperature & 298(2) K \\
\hline Wavelength & $0.71073 \AA$ \\
\hline Crystal system & Monoclinic \\
\hline Space group & $\mathrm{P} 2(1) / \mathrm{c}$ \\
\hline Cell dimensions & $\begin{array}{l}a=13.4824(16) \AA, b=9.1016(11) \AA, \\
c=11.4348(12) \AA, \beta=90.8170(10)\end{array}$ \\
\hline Volume & $871.56(18) \AA^{3}$ \\
\hline $\mathrm{Z}$ & 4 \\
\hline Density (calculated) & $1.318 \mathrm{mg} / \mathrm{m}^{3}$ \\
\hline Absorption coefficient & $0.088 \mathrm{~mm}^{-1}$ \\
\hline$F_{(000)}$ & 584 \\
\hline Index ranges & $-16 \leq \mathrm{h} \leq 14,-10 \leq \mathrm{k} \leq 9,-13 \leq 1 \leq 13$ \\
\hline Reflections collected/unique & $6863 / 2472\left[\mathrm{R}_{\mathrm{int}}=0.0548\right]$ \\
\hline Data/restraints/parameters & 2472 / 0 / 192 \\
\hline Goodness of fit indicator & 1.030 \\
\hline Final $R$ indices $[\mathrm{I}>2 \sigma(\mathrm{I})]$ & $\mathrm{R}_{1}=0.0468, \mathrm{wR}_{2}=0.1080$ \\
\hline $\mathrm{R}$ indices (all data) & $\mathrm{R}_{1}=0.0858, \mathrm{wR}_{2}=0.1371$ \\
\hline Largest diff. peak and hole & 0.299 and -0.229 e $\AA^{-3}$ \\
\hline
\end{tabular}

X-Ray structure determination: The single crystal of the title compound with approximate dimensions of $0.45 \mathrm{~mm}$ 
TABLE-2

SELECTED BOND LENGTHS $(\AA)$ AND ANGLES $\left({ }^{\circ}\right)$ FOR THE TITLE COMPOUND

\begin{tabular}{cccccc}
\hline Bond & Lengths & Bond & Lengths & Bond & Lengths \\
\hline $\mathrm{N}(1)-\mathrm{N}(2)$ & $1.377(3)$ & $\mathrm{O}(1)-\mathrm{C}(1)$ & $1.247(3)$ & $\mathrm{C}(2)-\mathrm{C}(11)$ & $1.466(3)$ \\
$\mathrm{N}(1)-\mathrm{C}(1)$ & $1.391(3)$ & $\mathrm{O}(2)-\mathrm{C}(11)$ & $1.224(3)$ & $\mathrm{C}(3)-\mathrm{C}(4)$ & $1.484(3)$ \\
$\mathrm{N}(1)-\mathrm{C}(5)$ & $1.418(3)$ & $\mathrm{C}(1)-\mathrm{C}(2)$ & $1.433(3)$ & $\mathrm{C}(5)-\mathrm{C}(10)$ & $1.371(4)$ \\
$\mathrm{N}(2)-\mathrm{C}(3)$ & $1.330(3)$ & $\mathrm{C}(2)-\mathrm{C}(3)$ & $1.381(3)$ & $\mathrm{C}(5)-\mathrm{C}(6)$ & $1.372(4)$ \\
$\mathrm{C}(6)-\mathrm{C}(7)$ & $1.375(4)$ & $\mathrm{C}(11)-\mathrm{C}(12)$ & $1.490(3)$ & $\mathrm{C}(14)-\mathrm{C}(15)$ & $1.372(4)$ \\
$\mathrm{C}(7)-\mathrm{C}(8)$ & $1.349(5)$ & $\mathrm{C}(12)-\mathrm{C}(17)$ & $1.384(3)$ & $\mathrm{C}(15)-\mathrm{C}(16)$ & $1.380(4)$ \\
$\mathrm{C}(8)-\mathrm{C}(9)$ & $1.370(5)$ & $\mathrm{C}(12)-\mathrm{C}(13)$ & $1.385(3)$ & $\mathrm{C}(16)-\mathrm{C}(17)$ & $1.382(4)$ \\
$\mathrm{C}(9)-\mathrm{C}(10)$ & $1.390(4)$ & $\mathrm{C}(13)-\mathrm{C}(14)$ & $1.380(3)$ & - & - \\
\hline Bond & Angles & $\mathrm{Bond}$ & $\mathrm{Angles}$ & $\mathrm{B}$ ond & Angles \\
\hline $\mathrm{N}(2)-\mathrm{N}(1)-\mathrm{C}(1)$ & $108.78(18)$ & $\mathrm{N}(2)-\mathrm{N}(1)-\mathrm{C}(1)$ & $108.78(18)$ & $\mathrm{C}(2)-\mathrm{C}(3)-\mathrm{C}(4)$ & $132.4(2)$ \\
$\mathrm{C}(3)-\mathrm{N}(2)-\mathrm{N}(1)$ & $109.26(19)$ & $\mathrm{C}(3)-\mathrm{N}(2)-\mathrm{N}(1)$ & $109.26(19)$ & $\mathrm{C}(10)-\mathrm{C}(5)-\mathrm{C}(6)$ & $120.1(2)$ \\
$\mathrm{O}(1)-\mathrm{C}(1)-\mathrm{N}(1)$ & $122.4(2)$ & $\mathrm{O}(1)-\mathrm{C}(1)-\mathrm{N}(1)$ & $122.4(2)$ & $\mathrm{C}(5)-\mathrm{C}(6)-\mathrm{C}(7)$ & $119.4(3)$ \\
$\mathrm{C}(3)-\mathrm{C}(2)-\mathrm{C}(1)$ & $107.3(2)$ & $\mathrm{C}(3)-\mathrm{C}(2)-\mathrm{C}(1)$ & $107.3(2)$ & $\mathrm{C}(8)-\mathrm{C}(7)-\mathrm{C}(6)$ & $121.6(3)$ \\
$\mathrm{C}(7)-\mathrm{C}(8)-\mathrm{C}(9)$ & $119.2(3)$ & $\mathrm{O}(2)-\mathrm{C}(11)-\mathrm{C}(12)$ & $119.9(2)$ & $\mathrm{C}(13)-\mathrm{C}(12)-\mathrm{C}(11)$ & $119.3(2)$ \\
$\mathrm{C}(8)-\mathrm{C}(9)-\mathrm{C}(10)$ & $120.6(3)$ & $\mathrm{C}(2)-\mathrm{C}(11)-\mathrm{C}(12)$ & $119.6(2)$ & $\mathrm{C}(14)-\mathrm{C}(15)-\mathrm{C}(16)$ & $119.9(3)$ \\
$\mathrm{C}(5)-\mathrm{C}(10)-\mathrm{C}(9)$ & $119.2(3)$ & $\mathrm{C}(17)-\mathrm{C}(12)-\mathrm{C}(13)$ & $118.6(2)$ & $\mathrm{C}(15)-\mathrm{C}(16)-\mathrm{C}(17)$ & $119.9(3)$ \\
$\mathrm{O}(2)-\mathrm{C}(11)-\mathrm{C}(2)$ & $120.5(2)$ & $\mathrm{C}(17)-\mathrm{C}(12)-\mathrm{C}(11)$ & $122.0(2)$ & $\mathrm{C}(16)-\mathrm{C}(17)-\mathrm{C}(12)$ & $120.7(2)$ \\
\hline
\end{tabular}

$\times 0.17 \mathrm{~mm} \times 0.14 \mathrm{~mm}$ was placed on a Bruker Smart 1000 diffractmeter equipped with Apex CCD area detector. The diffraction data were collected using a graphite monochromated $\mathrm{MoK}_{\alpha}$ radition $(\lambda=0.71073 \AA$ ) at $298(2) \mathrm{K}$. The structure was solved by using the program SHELXS-97 ${ }^{7}$ and Fourier difference techniques and refined by full-matrix least-squares method on $\mathrm{F}^{2}$ using SHELXL- $97^{8}$. Details of the data collection and refinements of the title compound are given in Table-1. The non-hydrogen atoms were refined anisotropically. Hydrogen atoms were added theoretically. CCDC: 894881.

The structure of the title compound is shown in Fig. 1. The dihedral angles formed by the pyrazolone ring with the two phenyl rings $\mathrm{C} 5-\mathrm{C} 10$ and $\mathrm{C} 12-\mathrm{C} 17$ are 28.36(3) and 59.31(5) ${ }^{\circ}$, respectively. Two carbonyl oxygen atoms are available for coordination with metals. The pyrazolone ring is planar and atoms $\mathrm{O} 1, \mathrm{~N} 1, \mathrm{~N} 2, \mathrm{C} 1, \mathrm{C} 2, \mathrm{C} 3, \mathrm{C} 4$ and $\mathrm{C} 11$ are almost coplanar, the largest deviation being 0.035 (3) $\AA$ for atom O1. But the O2 atom is not on the mean plane with a deviation of $0.652(2) \AA$. The dihedral angle between this mean plane and the pyrazolone ring of 1-phenyl-3-methyl-4-benzoylpyrazolon-5-one is $0.39(1)^{\circ}$. A strong intermolecular N2-H2 ‥O1 hydrogen bond (Table-2) stabilized the molecules into a 1D infinite chain parallel to the caxis (Fig. 2) ${ }^{9,10}$. This linkage is further stabilized by a pair of weak intermolecular C13-H13...O2 hydrogen-bonding interactions to form an infinite 2D supramolecular structure (Fig. 2) (Table-3) $)^{11,12}$.

\begin{tabular}{lcccc}
\multicolumn{5}{c}{ TABLE-3 } \\
\hline \multicolumn{1}{c}{ HYDROGEN-BONDING DATA $\left(\AA \AA^{\circ}\right)$} \\
\hline $\mathrm{N} 2-\mathrm{H} 2 \cdots \mathrm{O} 1$ & $\mathrm{~d}(\mathrm{D}-\mathrm{H})$ & $\mathrm{d}(\mathrm{H} \cdots \mathrm{A})$ & $\mathrm{d}(\mathrm{D} \cdots \mathrm{A})$ & $\angle \mathrm{D}-\mathrm{H} \cdots \mathrm{A}$ \\
$\mathrm{C} 13-\mathrm{H} 13 \cdots \mathrm{O} 2$ & 0.86 & 1.88 & $2.668(3)$ & 151 \\
& 0.93 & 2.55 & $3.288(3)$ & 137 \\
\hline
\end{tabular}

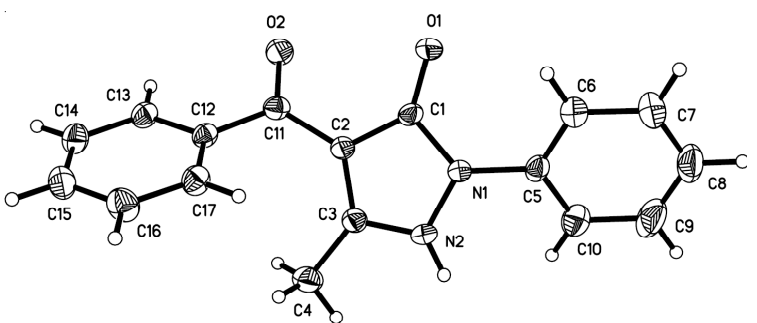

Fig. 1. Molecule structure of the title compound

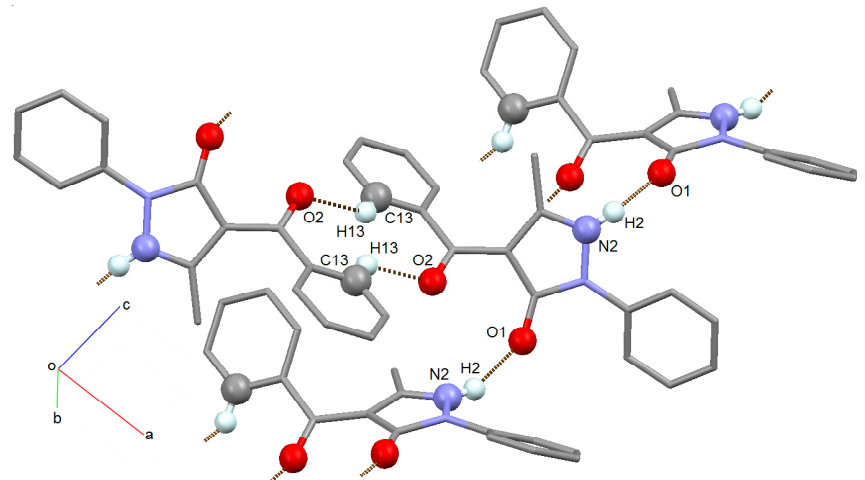

Fig. 2. View of the supramolecular structure within the title compound (hydrogen atoms, except those forming hydrogen bonds, are omitted for clarity)

\section{ACKNOWLEDGEMENTS}

This work was supported by the Foundation of Preparative Research of Jin-Chuan Corporation (No. 209125-1102, 1103).

\section{REFERENCES}

1. N.S. Karthikeyan, K.I. Sathiyanarayanan, P.G. Aravindan and P. Giridharan, Med. Chem. Res., 20, 81 (2011)

2. Z.Y. Yang, Synth. React. Inorg. Met.-Org. Nano-Met. Chem., 30, 1265 (2000).

3. W.K. Dong and J.G. Duan, J. Coord. Chem., 61, 781 (2008).

4. Q. Wang, Y. Wang and Z.Y. Yang, Chem. Pharm. Bull., 56, 1018 (2008).

5. S. Kumar and Sonika, Asian J. Chem., 19, 3869 (2007).

6. G.C. Xu, L. Zhang, L. Liu, G.F. Liu and D.Z. Jia, Thermochim. Acta, 429, 31 (2005).

7. G.M. Sheldrick, SHELXS97, Program for Crystal Structure Determination, University of Göttingen, Germany (1996).

8. G.M. Sheldrick, Acta Cryst., A64, 112 (2008).

9. W.K. Dong, Y.X. Sun, S.J. Xing, Y. Wang and X.H. Gao, Z. Naturforsch., 67b, 197 (2012).

10. W.K. Dong, J.G. Duan, L.Q. Chai, G.L. Liu and H.L. Wu, J. Coord. Chem., 61, 1306 (2008).

11. W.K. Dong, J.H. Feng and X.Q. Yang, Z. Kristallogr. NCS, 221, 447 (2006).

12. W.K. Dong, J.G. Duan, Y.H. Guan, J.Y. Shi and C.Y. Zhao, Inorg. Chim. Acta, 362, 1129 (2009). 